\title{
RGB Demultiplexer Based on Multicore Polymer Optical Fiber
}

\author{
Rami Dadabayev ${ }^{1}$, Moshe $\operatorname{ran}^{2}$ and Dror Malka ${ }^{1,2 *}$ \\ ${ }^{1}$ MostlyTek LTD , Modiin, Israel \\ ${ }^{2}$ Faculty of Engineering, Holon Institute of Technology (HIT) \\ Holon 5810201, Israel \\ * Correspondence: drorm@ @it.ac.il
}

\begin{abstract}
Wavelength division multiplexing (WDM) is a good solution for increasing data bitrate communication of multicore polymer optical fiber (MC-POF) based visible light communication (VLC) system. However, this solution requires adding more optical components to the system which can limit its performance. In order to solve this issue, we propose a new design for an RGB demultiplexer based on polycarbonate (PC) MC-POF structure. The new structure is based on replacing several air-holes areas with PC layers over the fiber length which enables controlling the light propagation direction between the pc layers. The positions of the PC layers and the key geometrical parameters of the MC-POF were optimized and analyzed utilizing the beam propagation method (BPM). Results show that an RGB wavelength splitter can be obtained over a light propagation of $20 \mathrm{~mm}$ with an excellent crosstalk of -19.436 to 26.474 dB, low losses of 0.901 to $1.246 \mathrm{~dB}$ and a large bandwidth of 5.6 to $11.3 \mathrm{~nm}$.
\end{abstract}

Keywords-VLC; demultiplexer; MC-POF; ВPM.

\section{INTRODUCTION}

Polymer optical fiber (POF) is used as an excellent replacement for the traditional glass fiber for short distances. Polymer fiber consists of polymer core that can transmit the signal as efficiently as glass core, the core is surrounded with cladding that can be made of silica, another polymer or perfluorinated material.

Polymer fibers can be used in cars, buildings and automation systems [1]. POF has several advantages such as robustness to mechanical stress and electromagnetic interference, easy installation, low cost connectors and low-price systems. Another variation of the POF is the multicore POF (MC-POF) that consists from many cores surrounded in a sea of cladding, those fibers have much resemblance to the multicore photonic crystal fibers [2-3]. MC-POF utilize the photonic bandgap alongside to the modified total internal reflection mechanisms allowing design of new compact optical components [4]. The transmission bitrate of the MC-POF can reach up to $10.7 \mathrm{~Gb} / \mathrm{s}$ for short distances [5].

To further increase the transmission bitrate in VLC systems, Wavelength division multiplexing (WDM) techniques can be utilized. WDM technique allows combining several signals together and transmits them simultaneously while each signal is carried by a different wavelength. According to the latest researches, the system would be able to support the transmission bitrate of up to $8 \mathrm{~Gb} / \mathrm{s}$ [6-8] and even a higher transmission bitrate is possible [9-10]. WDM systems are composed of several components, one of them is the demultiplexer, it allows to separate the signal carried by different wavelengths from one input to several outputs. Using demultiplexer gives a lot of benefits such as high data bitrate transmission, low propagation bitrate and low bit error rate [11$12]$.

Visible light communication (VLC) is an alternative to the widely common radio frequency communication and has a growing interest among many researchers in the past several years due to the development of the new high performance white LED [13]. Compared to the radio frequency communication, VLC has many advantages and useful properties, among them: unregulated bandwidth, high transmission capacity with low radiation rates [14], unaffected by electromagnetic interference [15], allow establishing secure communication systems [16]. VLC technology is widely studied and there are a variety of published novel ideas [17].

Polycarbonate (PC) is an amorphous thermoplastic material with exceptionally high impact strength, excellent transparency for visible light [18], high dimensional stability, and moderate temperature resistance. PC has a glass transition temperature of $148^{\circ} \mathrm{C}$, high gloss finishing, low mold shrinkage, low moisture absorption properties, good insulation resistance properties, alongside dielectric constant that is independent of temperature. All these properties make the PC a very suitable material for microstructure POF [19].

The proposed RGB demultiplexer is composed of integrated PC layers along the fiber length and based on controlling the light coupling between adjacent channels.

In this paper, a new design of RGB wavelength demultiplexer based on PC MC-POF is introduced.

Numerical investigations were carried out on the geometrical parameters of the PC MC-POF structure in order to find the optimal coupling length sizes that are most suitable for the RGB operated wavelengths.

The optimizations and simulations were performed using the 
beam propagation method (BPM) alongside with Matlab codes [20].

The main benefit of this design is that the PC MC-POF can function as a demux device without additional bulk components that current commonly used today [21-23]. This can lead to a compact WDM system that can be utilized to obtain better performances.

\section{RGB DEMULTIPLEXER STRUCTURE AND THEORETICAL ASPECT}

The proposed RGB demultiplexer is based on PC MC-POF hexagonal structure. In this structure, the background material is CYTOP composed with air-holes and PC layers along the fiber length. The physical mechanism of the RGB demultiplexer is based on light confinement inside the core and controlling the coupling length size between the neighboring cores.

PC was chosen as an excellent material for transmitting light due to its low absorption in the visible range.

The POF structure is based on one input channel, air-holes layers, PC layers and three output channels as shown in Figures 1(a)-(b). The POF geometric parameters, locations of the airholes and PC areas were optimized to allow an efficient light coupling for the operating signals while filtering other wavelengths to obtain the best insulation between the channels. In figures $1(a-b)$, the length of the fiber is denoted as $z, \Lambda$ (pitch) denotes the distance between two neighboring air holes, $\mathrm{d}$ denotes the diameter of the air-holes, $\mathrm{d} / \Lambda$ denotes air holes diameter to pitch ratio. The values of the POF geometrical parameters are:

$\mathrm{z}=20 \mathrm{~mm}, \mathrm{~d}=0.241637 \mu \mathrm{m}, \Lambda=0.5498 \mu \mathrm{m}, \mathrm{d} / \Lambda=0.4395$.

Figure 1(a) shows a cross-section of the RGB demultiplexer structure in $\mathrm{x}-\mathrm{z}$ plane at $\mathrm{y}=0 \mathrm{~mm}$. In this figure, the red areas denote PC, beige areas denote CYTOP and the light blue areas denote air.

Figure 1(b) shows a cross-section of the RGB demultiplexer structure in the $x-y$ plane at the output $(z=20 \mathrm{~mm})$.

The crosstalk is given by [24]:

$$
\text { C. } T_{n}=\frac{1}{2} \sum_{m=1}^{3} 10 \log \left(\frac{P_{m}}{P_{n}}\right)(d b)
$$

Where Pn is the power from the transmission channel and Pm is the interference power from the neighboring channels. Insertion loss is given by [24]:

$$
\text { Loss }=-10 \log \left(\frac{P_{\text {out }}}{P_{\text {in }}}\right)(\mathrm{db})
$$

Where Pout is the power at the output channel and Pin is the input power at channel 2 .

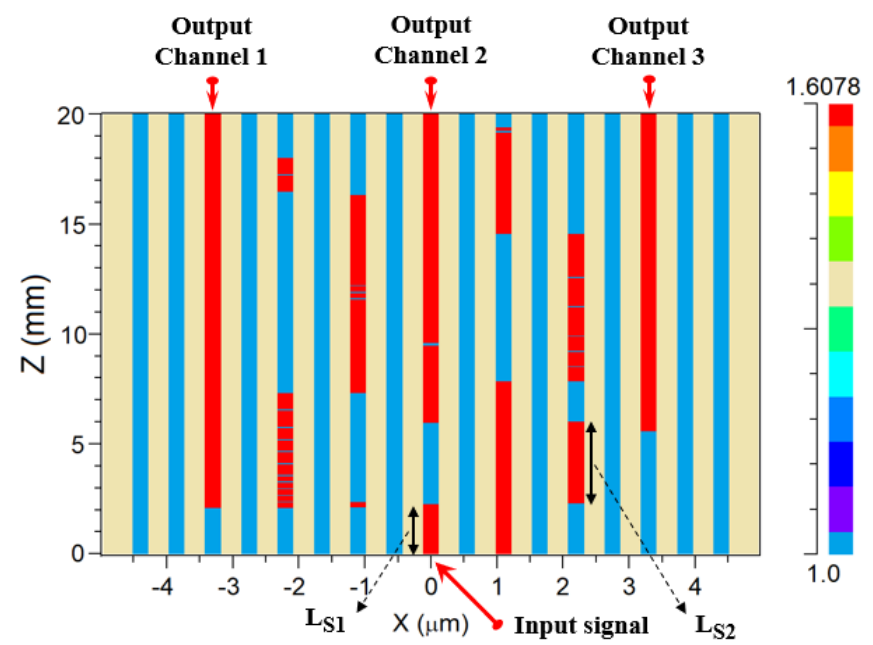

(a)
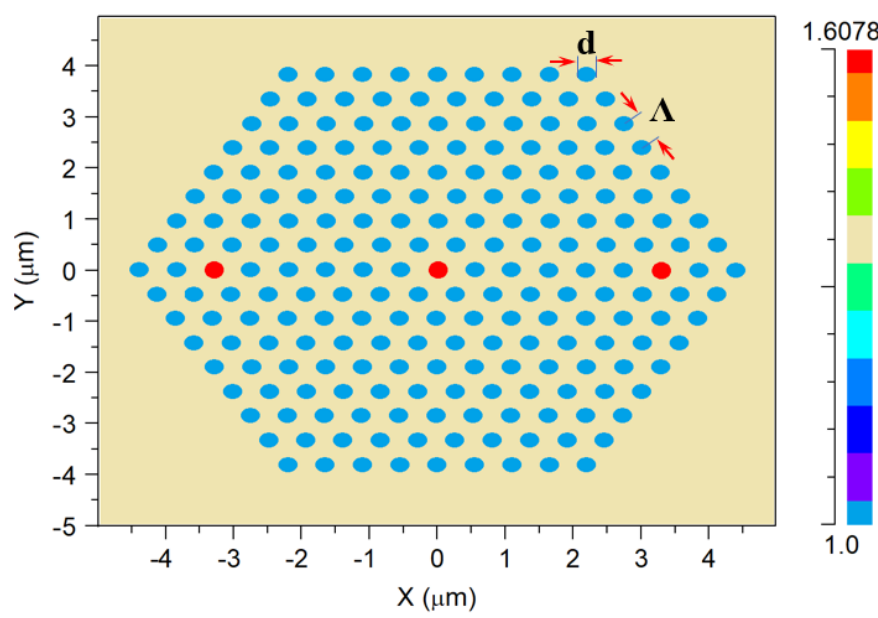

(b)

Fig. 1. Refractive index profile of the RGB wavelength demultiplexer for $450 \mathrm{~nm}$ wavelength: (a) $\mathrm{x}-\mathrm{z}$ plane at $\mathrm{y}=0$ $\mathrm{mm}$; (b) $\mathrm{x}-\mathrm{y}$ plane at $\mathrm{z}=20 \mathrm{~mm}$.

Coupling length can be found using the following equation [25]:

$$
\mathrm{L}_{\text {Coupling }}^{\lambda}=\frac{\pi}{\mathrm{k}_{0}\left(\mathrm{n}_{\text {symmetric }}(\mathrm{d}, \Lambda, \lambda)-\mathrm{n}_{\text {anti-symmertric }}(\mathrm{d}, \Lambda, \lambda)\right)}
$$

Where $\mathrm{k} 0$ is the free space vector, nanti-symmetric is the antisymmetric effective refractive index and nsymmetric is the symmetric effective refractive index.

A better way to find the coupling length is by numerical analysis of the transfer energy between two adjacent PC cores using BPM.

It is worth mentioning that light can be coupled only between adjacent $\mathrm{PC}$ cores and the conditions for dividing between the RGB wavelengths in the PC MC-POF are given by: 


$$
\begin{aligned}
& \mathrm{L}_{\mathrm{S} 1} \approx \mathrm{p}_{1} \mathrm{~L}_{\text {Coupling }}^{\lambda_{\text {Red }}}=\left(\mathrm{p}_{1}+\mathrm{q}_{1}\right) \mathrm{L}_{\text {Coupling }}^{\lambda_{\text {Blue, }} \lambda_{\text {Green }}} \\
& \mathrm{L}_{\mathrm{S} 2} \approx \mathrm{p}_{2} \mathrm{~L}_{\text {Coupling }}^{\lambda_{\text {Blue }}}=\left(\mathrm{p}_{2}+\mathrm{q}_{2}\right) \mathrm{L}_{\text {Coupling }}^{\lambda_{\text {Green }}}
\end{aligned}
$$

Where $\mathrm{L}_{\mathrm{S} 1}$ is the PC layer located at the input channel 2 and $\mathrm{L}_{\mathrm{S} 2}$ is the PC layer located between channel 2 and channel 3, $\mathrm{p}_{1 / 2 / 3}$ is a natural number, and $\mathrm{q}_{1 / 2 / 3}$ is an odd number, $\mathrm{L}_{\text {Coupling }}^{\lambda}$ is the coupling length of the chosen wavelength.

$\mathrm{L}_{\mathrm{S} 1}$ and $\mathrm{L}_{\mathrm{S} 2}$ are functioning as an optical coupler switches that control the light propagation direction and direct the signals to the appropriate channel according to their RGB wavelengths. The other PC layers locations were optimized in order to obtain a good isolation between the three channels.

\section{SimUlation RESUltS}

The simulations and the numerical investigations of the PC MC-POF structure were performed by RSoft Photonics CAD Suite software that utilized the BPM. Furthermore, the BPM results were analyzed using Matlab scripts codes in order to optimize the demultiplexer parameters for best performances.

Figure 2 shows the intensity profile of the energy transfer between two adjacent PC cores over the fiber length (z-axis) for $530 \mathrm{~nm}$ operated wavelength. the figure also shows the sinusoidal behavior of the oscillations between the two adjacent PC cores over the light propagation direction.

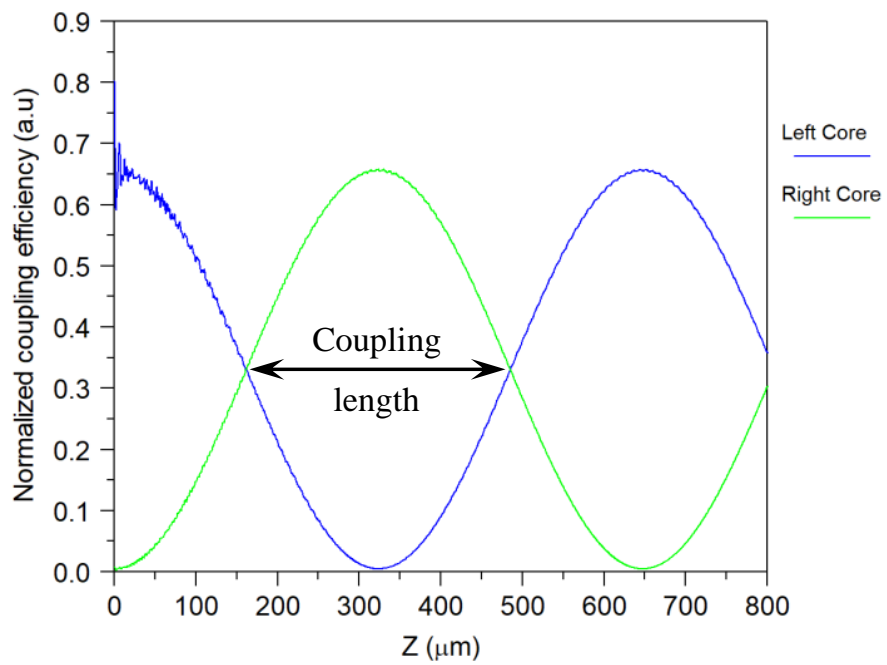

Fig. 2. Normalized coupling efficiency as function of light propagation (z-axis) for $530 \mathrm{~nm}$ wavelength.

It can be noticed form figure 2 that the coupling length size can be found by calculating the distance between the intersection between the two adjacent PC cores.

By performing the numerical analysis as shown in figures 2 for each wavelength in the visible range spectrum it can be possible to extract the coupling length data for each wavelength.

From the coupling length data, the most suitable operating wavelengths to match the coupling length of the POF demultiplexer structure can be found.

Signal demultiplexing is obtained by utilizing the switches $\mathrm{L}_{\mathrm{S} 1}$ and $L_{S 2}$. The optimal lengths of the switches can be found by extracting the coupling length from the data for the chosen RGB signals and solving equation (4).

The chosen operated wavelengths are: $\lambda 1=600 \mathrm{~nm}$ for channel 1 (Red), $\lambda 2=450 \mathrm{~nm}$ for channel 2 (Blue) and $\lambda 3=530 \mathrm{~nm}$ for channel 3 (Green). The coupling length sizes of the RGB channels are 123.24, 324.04, $1988.6 \mu \mathrm{m}$, respectively. Switches $\mathrm{L}_{\mathrm{S} 1}$ and $\mathrm{L}_{\mathrm{S} 2}$ lengths are 2.29 and $3.74 \mathrm{~mm}$ respectively.

Table 1 shows the refractive index values of CYTOP and PC [26] for the chosen operating wavelengths.

TABLE I. RGB DEMUX MATERIALS REFRACTIVE INDEX VALUES.

\begin{tabular}{c|ccc}
\hline$\lambda(\mathrm{nm})$ & $\mathbf{4 5 0}$ & $\mathbf{5 3 0}$ & $\mathbf{6 0 0}$ \\
\cline { 2 - 4 } $\mathrm{n}_{\text {СYTOP }}$ & 1.344 & 1.34 & 1.3396 \\
$\mathrm{n}_{\text {PC }}$ & 1.6078 & 1.592 & 1.5835 \\
\hline
\end{tabular}

Figure 3 shows the optimal geometrical parameters $(d / \Lambda)$ of the MC-POF structure for the RGB wavelengths.

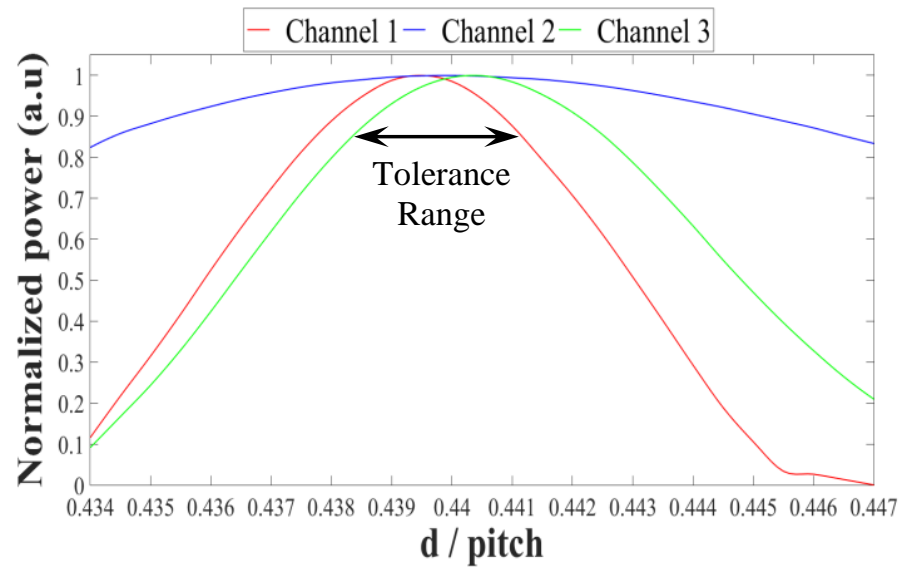

Fig. 3. Normalized power as a function of the geometrical parameters of the multicore MC-POF $(d / \Lambda)$ for the RGB wavelengths.

It can be seen from figure 3 that the optimal value is 0.4395 for the operating wavelengths. In addition, the tolerance range was analyzed in order to fulfill the fabrication requirement. As seen in the figure the lower range is limited by channel 1 (red) and the higher range is limited by channel 3 (green), the optimal tolerance range of $\mathrm{d} / \Lambda$ is between $0.4383-0.4412$. This range ensures output power above $85 \%$ for all the chosen operated 
wavelengths.

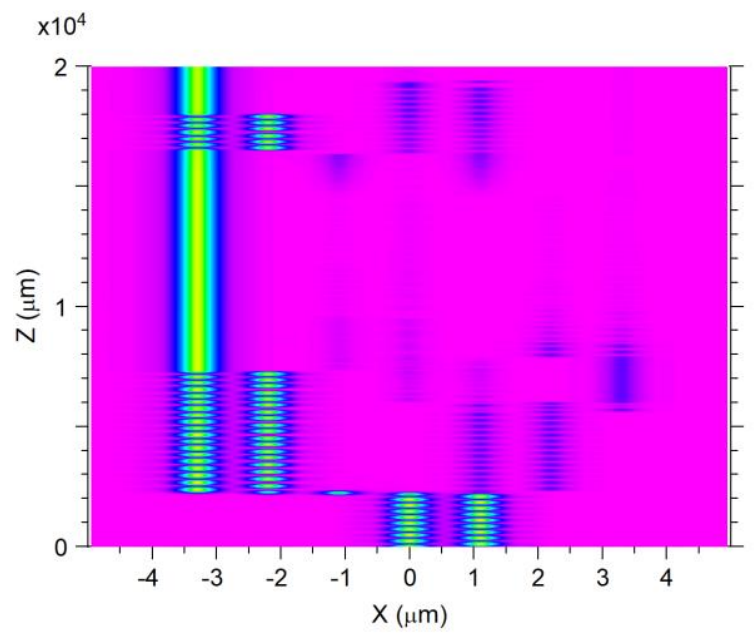

(a)
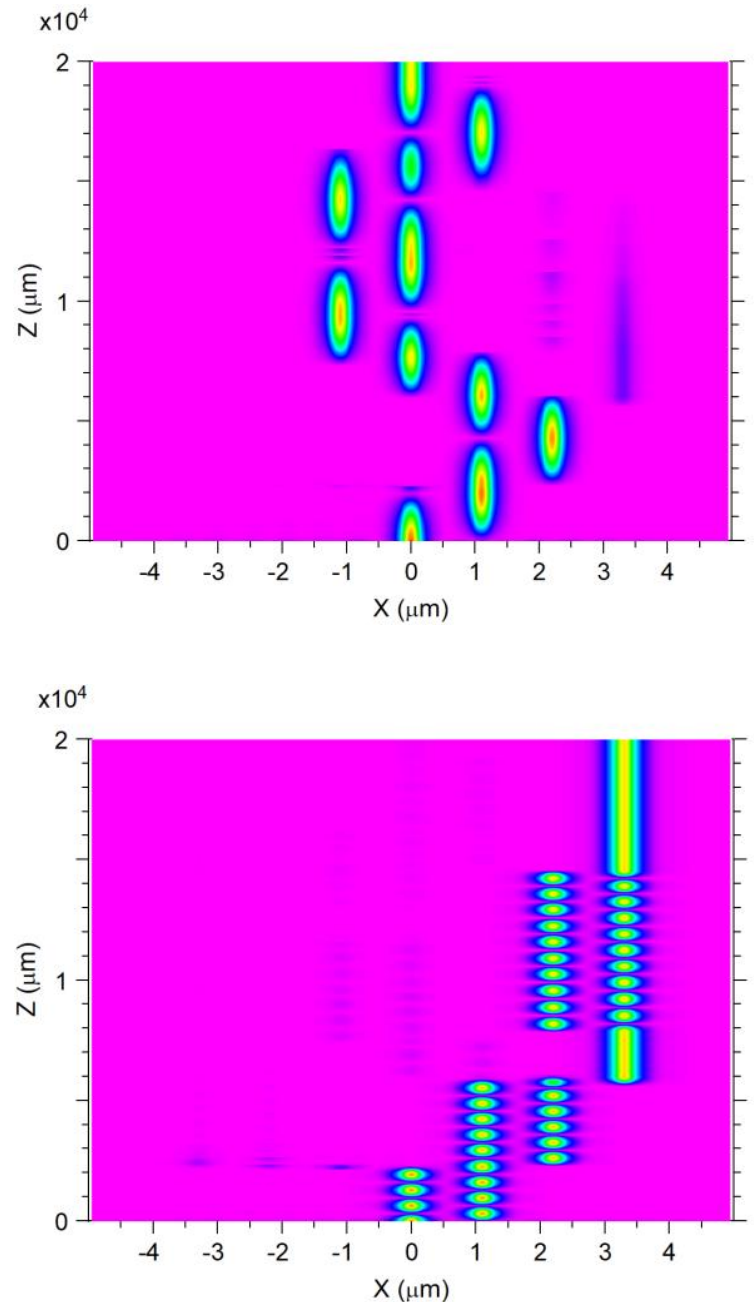

(b)

(c)

Fig. 4. Intensity profile of the RGB wavelength demultiplexer at X-Z plane. (a) Channel 1 (red), $\lambda=600 \mathrm{~nm}$; (b) Channel
2 (blue), $\lambda=450 \mathrm{~nm}$; (c) Channel 3 (green), $\lambda=530 \mathrm{~nm}$. Figures 4(a)-(c) show the intensity light propagation over the fiber length at x-z plane for the RGB wavelengths and the light coupling between $\mathrm{PC}$ neighboring cores.

Figure 4(a) shows the optical path for the red light $(\lambda=600$ $\mathrm{nm}$ ) from input channel 2 to the output of channel 1. Coupler switch $\mathrm{L}_{\mathrm{S} 1}$ directed the red light towards channel 1 at $\mathrm{z}=2.29$ $\mathrm{mm}$; the light is coupling and oscillated between neighboring PC cores over $z$ ranges of 0-2.29 mm, 2.29-7.3 mm and 16.5$17.24 \mathrm{~mm}$; the red light is confined inside channel 1 over $\mathrm{z}$ ranges of 7.3-16.5 $\mathrm{mm}$ and $17.24-20 \mathrm{~mm}$.

Figure 4(b) shows the optical path for the blue light $(\lambda=450$ nm) from input channel 2 to the output of channel 2. Coupler switch $\mathrm{L}_{\mathrm{S} 1}$ at $\mathrm{z}=2.29 \mathrm{~mm}$ directed the blue light towards coupler switch $\mathrm{L}_{\mathrm{S} 2}$ and then LS2 directed the light towards channel 2 at $\mathrm{z}=6.04 \mathrm{~mm}$; the light is coupling and oscillated between neighboring PC cores over $\mathrm{z}$ ranges of $0-2.29 \mathrm{~mm}$, 2.3-6.04 mm, 9.6-19.4 mm; the blue light is confined inside channel 2 over $\mathrm{z}$ range of $19.4-20 \mathrm{~mm}$.

Figure 4(c) shows the optical path for the green light $(\lambda=530$ $\mathrm{nm}$ ) from input channel 2 to the output of channel 3. Coupler switch $\mathrm{L}_{\mathrm{S} 1}$ at $\mathrm{z}=2.29 \mathrm{~mm}$ directed the green light towards coupler switch $\mathrm{L}_{\mathrm{S} 2}$ and then $\mathrm{L}_{\mathrm{S} 2}$ directed the light towards channel 3 at $\mathrm{z}=6.04 \mathrm{~mm}$; the light is coupling and oscillated between neighboring PC cores over $\mathrm{z}$ ranges of 0-6.04 mm, $7.95-14.55 \mathrm{~mm}$; the green light is confined inside channel 3 over $\mathrm{z}$ range of $14.55-20 \mathrm{~mm}$.

It is important to emphasize that the locations of the PC layers over the fiber length were optimized in order to obtain better performances for the operated RGB wavelengths.

Results show that the overall fiber length size of the PC MCPOF is only $20 \mathrm{~mm}$ which means that the proposed device has a compact size compering to the RGB demux based on photonic crystal fiber [27].

Figure 5 shows the transmission for the RGB wavelengths and it can be noticed that a good transmission can be obtained.

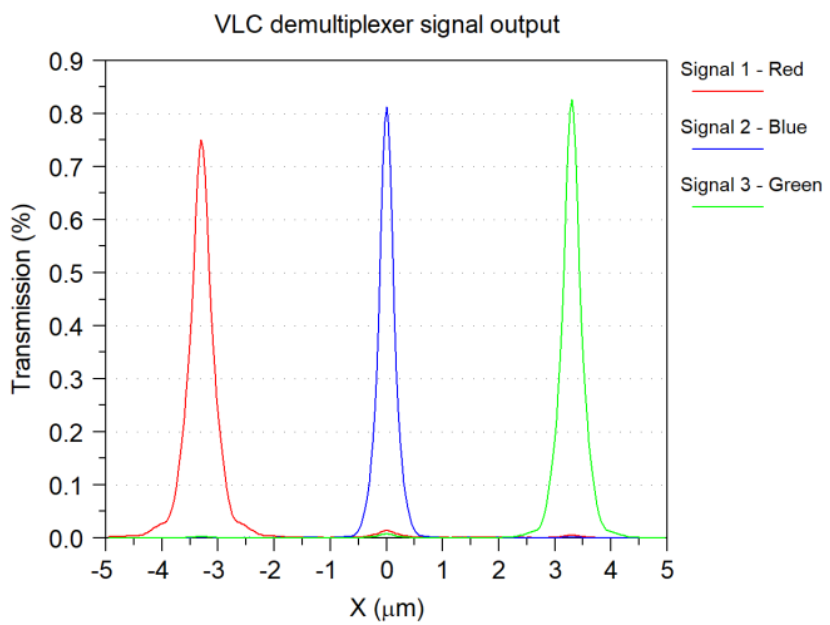

Fig. 5. Transmission for RGB wavelengths. 
In addition, a good insulation between the RGB channels is obtained as shown in figure 5 which means that the interferences between channels are very low.

By using the data obtained from figure 5 and combined with solving equations (1) and (2), crosstalk, full width at half maximum (FWHM) and insertion loss for the RGB wavelengths can be found. Table 2 shows the values of crosstalk, FWHM, and loss for each one of the RGB wavelengths.

TABLE II. VALUES OF CROSSTALK, FWHM, AND LOSS FOR THE OPERATING WAVELENGTHS.

\begin{tabular}{c|ccc}
\hline$\lambda(\mathrm{nm})$ & $\mathbf{4 5 0}$ & $\mathbf{5 3 0}$ & $\mathbf{6 0 0}$ \\
\cline { 2 - 4 } Crosstalk $(\mathrm{dB})$ & -26.471 & -22.704 & -19.436 \\
FWHM $(\mathrm{nm})$ & 11.3 & 8.2 & 5.6 \\
Loss $(\mathrm{dB})$ & 0.901 & 0.828 & 1.246 \\
\hline
\end{tabular}

\section{CONCLUSION}

In this paper, a new design of visible light RGB demultiplexer based on PC MC-POF was demonstrated.

This study shows the benefices of using PC as the core material in the MC-POF and the ability to control the light propagation direction inside the microstructure by a variation of the operating wavelength.

Simulation results show that the RGB wavelengths can propagate inside the device and to demultiplexed optimally into three channels after a distance of $20 \mathrm{~mm}$ with low insertion loss between the range of 0.901 to $1.246 \mathrm{~dB}$.

In addition, the proposed device has low crosstalk in the range of -19.436 to $-26.474 \mathrm{~dB}$ and a large optical bandwidth range of 5.6 to $11.3 \mathrm{~nm}$.

By analysis the numerical results, it is clear that the main benefit is that PC MC-POF design does not require additional components in order to act as RGB demultiplexer. This benefit can be utilized to design new and compact VLC-WDM system that can be used to obtain better performances comparing to VLC-WDM system that used bulk components.

Therefore, this device can be integrated into WDM technology without adding additional components. This can be very useful to improve the performance of VLC systems and especially for increasing data bitrate transmission.

\section{ACKNOWLEDGMENTS}

The authors gratefully acknowledge the financial support of the EU Horizon 2020 program towards the Internet of Radio -Light project H2020 -ICT761992.

\section{REFERENCES}

[1] I. Mollers, D. Jager, R. Gaudino, A. Nocivelli, H. Kragl, O. Ziemann, N. Weber, T. Koonen, C. Lezzi, A. Bluschke, S. Randel, "Plastic Optical Fiber Technology for Reliable Home Networking: Overview and Results of the EU Project POF-ALL", IEEE Communications Magazine, vol. 47, issue. 8, pp. 58-68, AUG 2009.

[2] D. Malka, A. Peled, "Power splitting of $1 \times 16$ in multicore photonic crystal fibers", Elsevier - Applied Surface Science, vol. 412, pp. 34-39, FEB 2017

[3] D. Malka, Y. Sintov, Z. Zalevsky, "Fiber-laser monolithic coherent beam combiner based on multicore photonic crystal fiber", Optical Engineering 54(1), 011007, SEP 2014.

[4] A. Barh, R.K. Varshney, B.P. Pal, G.P. Agrawal, B M.A. Rahman, "Design of a Polymer-Based Hollow-Core Bandgap Fiber for Low-Loss Terahertz Transmission", IEEE Photonics Technology Letters, vol. 28, issue. 15, pp. 1703-1706, AUG 2016.

[5] S. Loquai, R. Kruglov, C.A. Bunge, O. Ziemann, B. Schmauss, J. Vinogradov, "10.7-Gb/s Discrete Multitone Transmission Over 25-m Bend-Insensitive Multicore Polymer Optical Fiber", IEEE Photonics Technology Letters, vol. 22, issue 21, pp. 1604-1606, Nov 2010.

[6] Y. Wang, L. Tao, X. Huang, J. Shi, N. Chi, "Enhanced Performance of a High-Speed WDM CAP64 VLC System Employing Volterra SeriesBased Nonlinear Equalizer", IEEE Photonics Journal, vol. 7, issue 3, JUN 2015.

[7] Y. Wang, L. Tao, X. Huang, J. Shi, N. Chi, "8-Gb/s RGBY LED-Based WDM VLC System Employing High-Order CAP Modulation and Hybrid Post Equalizer", IEEE Photonics Journal, vol. 7, issue 6, DEC 2015.

[8] K. Liang, C.W. Chow, Y. Liu, "RGB visible light communication using mobile-phone camera and multi-input multi-output", Optics Express, vol 24, issue 9, pp. 9383-9388, APR 2016.

[9] T.C. Wu, Y.C. Chi, H.Y. Wang, C.T. Tsai, Y.F. Huang, G.R. Lin, "Tricolor R/G/B Laser Diode Based Eye-Safe White Lighting Communication Beyond $8 \mathrm{Gbit} / \mathrm{s}$ ", Scientific Reports, vol. 7, 11, JAN 2017.

[10] L.Y. Wei, C.W. Hsu, C.W. Chow, C.H. Yeh, "20.231 Gbit/s tricolor $\mathrm{red} / \mathrm{green} / \mathrm{blue}$ laser diode based bidirectional signal remodulation visible-light communication system", Photonics Research, vol. 6, issue 5, pp. 422-426, APR 2018.

[11] Charlier J., Laurent B., Berlioz P., Perbos J.L., "Multi/demultiplexer and spectral isolator for optical intersatellites communications", Proc. SPIE Opt. Space Commun. vol. 1131, OCT 1989.

[12] Debashis De, "Basic Electronics", Dorling Kindersley, 2010.

[13] A.M. Khalid et al., "1-Gb/s Transmission Over a Phosphorescent White LED by Using Rate-Adaptive Discrete Multitone Modulation," IEEE Photonics Journal, vol. 4, no. 5, pp. 1465-1472, 2012.

[14] H. Le Minh, et al, " $100 \mathrm{Mb} / \mathrm{s}$ NRZ visible light communications using a post equalized white LED”, IEEE Photon. Technol. Lett., Vol. 21, pp. 1063-1065, AUG 2009.

[15] W. Chowa, et al. "Secure communication zone for white-light LED visible light communication", Optics Communications vol. 344, pp. 8185, JUN 2015.

[16] S.H. Yang, D.H. Kwon, S.K. Han., "Implementation of differentiated services in indoor visible-light communication using interchannel interference", Optics Communications, vol. 385, pp. 113-117, FEB 2017.

[17] S.H. Chen, C.W Chow, "Color-filter-free spatial visible light communication using RGB-LED and mobile phone camera", Optics Express, vol 22, issue 25, DEC 2014.

[18] O. Ziemman, J. Krauser, P.E. Zamzow, and W. Daum, "Optical Fibers," in POF Handbook. Optical Short Range Transmission Systems, 2nd ed., Ed. Berlin: Springer, 2008, pp. 37-231.

[19] M.A. van Eijkelenborg, A. Argyros, and S.G. Leon-Saval, "Polycarbonate hollow-core microstructured optical fiber," Opt. Lett., vol. 33, no. 21, pp. 2446-2448, 2008. 
[20] C.L. Xu; et al, "A full vectorial beam propagation method for anisotropic waveguides", Lightwave Technology, vol. 12, pp. 1926, NOV 1994.

[21] R. Kruglov, J. Vinogradov, O. Ziemann, S. Loquai, C. A. Bunge, "10.7Gb/s Discrete Multitone Transmission Over 50-m SI-POF Based on WDM Technology", IEEE PHOTONICS TECHNOLOGY LETTERS, vol. 24, issue. 18, pp. 1632-1634, SEP 2012.

[22] M. Jon `ci'c, R. Kruglov, M. Haupt, R. Caspary, J. Vinogradov, U. H. P. Fischer, "Four-Channel WDM Transmission Over 50-m SI-POF at $14.77 \mathrm{~Gb} / \mathrm{s}$ Using DMT Modulation", IEEE PHOTONICS TECHNOLOGY LETTERS, vol. 26, issue. 13, pp. 1328-1331, JUL 2014.

[23] P. J. Pinz'on, I. P. Garcil'opez, C. V'azquez, "Efficient Multiplexer / Demultiplexer for Visible WDM Transmission over SI-POF Technology”, JOURNAL OF LIGHTWAVE TECHNOLOGY, vol. 33, issue 17, pp. 3711-3718, SEP 2015.

[24] B.B.B. Zaken; T. Zanzury. D. Malka, "An 8-Channel Wavelength MMI Demultiplexer in Slot Waveguide Structures", Materials, vol. 9, pp. 881, NOV 2016.

[25] D. Malka, G. Katz, "An Eight-Channel C-Band Demux Based on Multicore Photonic Crystal Fiber", Nanomaterials, vol. 8, issue. 845, OCT 2018.

[26] N. Sultanova, S. Kasarova and I. Nikolov, "Dispersion properties of optical polymers", Acta Physica Polonica, vol. A 116, pp. 585-587, 2009.

[27] R. Dadabayev, N. Shabairou, Z. Zalevsky, D. Malka, “A visible light RGB wavelength demultiplexer based on silicon-nitride multicore PCF", Elsevier - Optics and Laser Technology, vol. 111, pp. 411-416, OCT 2018. 